Article

\title{
Comparison of Horizontal Accuracy, Shape Similarity and Cost of Three Different Road Mapping Techniques
}

\author{
Hyeongkeun Kweon ${ }^{1,2}\left(\right.$, Myeongjun Kim ${ }^{3}$, Joon-Woo Lee ${ }^{1}$, Jung Il Seo ${ }^{4}(\mathbb{D}$ and \\ Hakjun Rhee ${ }^{1, *(D)}$ \\ 1 Department of Environment and Forest Resources, Chungnam National University, 99 Daehak-ro, \\ Yuseong-gu, Daejeon 34134, Korea; hkkweon00@gmail.com (H.K.); jwlee@cnu.ac.kr (J.-W.L.) \\ 2 School of Environmental and Forest Sciences, University of Washington, Seattle, WA 98195-2100, USA \\ 3 Forest Environment \& GeoSpatial Technology Research Institute, Daonchae B101, 6 Jijokbuk-ro, Yuseong-gu, \\ Daejeon 34070, Korea; festri.kim@gmail.com \\ 4 Department of Forest Resources, Kongju National University, 54 Daehak-ro, Yesan-eup, Yesan-gun, \\ Chungcheongnam-do 32439, Korea; jungil.seo@kongju.ac.kr \\ * Correspondence: hakjun.rhee@gmail.com; Tel.: +82-10-4899-8751
}

Received: 3 April 2019; Accepted: 22 May 2019; Published: 24 May 2019

\begin{abstract}
Accurate spatial information on forest roads is important for forest management and harvest operations. This study evaluated the positional accuracy, shape similarity, and cost of three mapping techniques: GNSS (Global Navigation Satellite System) mapping, CAD file conversion (as-built drawing), and image warping. We chose five road routes within the national forest road system in the Republic of Korea and made digital road maps using each technique. We then compared map accuracy to reference maps made from field surveys. The mapping and field-survey results were compared using point-correspondence, buffering analysis, shape index, and turning function methods. The comparisons indicate that GNSS mapping is the best technique because it generated the highest accuracy (Root Mean Square Error: GNSS mapping 1.28, image warping 7.13, CAD file conversion 13.35), the narrowest buffering width for $95 \%$ of the routes overlapped (buffering width: GNSS mapping $1.5 \mathrm{~m}$, image warping $18 \mathrm{~m}$, CAD file conversion $24 \mathrm{~m}$ ), highest shape similarity (shape index: GNSS mapping 19.6-28.9, image warping 7.2-10.8, CAD file conversion 6.5-7.4), and smallest area size difference in turning function analysis (GNSS mapping 2814-4949, image warping 7972-26,256, CAD file conversion 8661-27,845). However, GNSS requires more time $(236 \mathrm{~min} / \mathrm{km})$ and costs more $(\$ 139.64 / \mathrm{km})$ to produce a digital road map as compared to CAD file conversion (99 $\mathrm{min} / \mathrm{km}$ and $\$ 40.90 / \mathrm{km}$ ) and image warping (180 $\mathrm{min} / \mathrm{km}$ and $\$ 81.84 / \mathrm{km})$. Managers must decide on the trade-off between accuracy and cost while considering the demand and purpose of maps. GNSS mapping can be used for small-scale mapping or short-haul routes that require a small error range. Image warping was the lowest cost and produced low-accuracy maps, but may be suitable for large-scale mapping at the regional or national level. CAD file conversion was expected to be the most accurate method, because it converted as-built drawings to a map. However, we found that it was the least accurate method, indicating low accuracy of the as-built drawings. Efforts should be made to improve the accuracy of the as-built drawings in Korea.
\end{abstract}

Keywords: road network; mapping accuracy; GIS; total station; GNSS; as-built drawing; image warping

\section{Introduction}

Forest roads are the infrastructure that affects accessibility to work sites that allow the continuation of forest management and harvest operations [1,2]. Forest roads are often considered an integral part 
of forest operations and, therefore, existing and new roads are either mapped or planned within the entire harvest operations platform. Because roads are essential for equipment and material movement, accurate spatial information on forest roads is crucial.

Forest maps are the most commonly used method to present spatial information. They can show terrain, land use, route position, land ownership, and stream channel networks. The display of this information requires current maps that are highly accurate [3,4]. Recently, with the advancement of computer technology, Geographic Information System (GIS) is widely used to analyze spatial information. In addition, thematic maps of forest vegetation, soil, and topography are now available in GIS [5]. In particular, GIS software is used to plan forest management activities that require a road network for planning and conducting forest operations [6]; necessitating a digital mapping system that is both accurate and cost-effective [2].

Spatial information for forest road mapping can be obtained in four ways: (i) digitizing after scanning of non-digital data (i.e., paper maps and as-built drawings); (ii) aerial photography and satellite imagery; (iii) field surveying; and (iv) data transference [7]. First, digitizing after scanning non-digital maps is highly efficient because it uses existing non-digital data. However, this type of mapping is impossible when non-digital data do not exist. Furthermore, periodic updates of non-digital data are required to maintain accuracy even if they do exist [4] and digitizing has the potential for errors caused by scaling or by the operator [7]. Second, aerial photography and satellite imagery are currently the most frequently-used methods for acquisition of spatial information due to their accuracy and efficiency, but it is expensive to obtain this data [8]. Third, field surveying is a time-consuming task and it is difficult to map a wide range of sites because data acquisition is performed by a few surveyors [6]. Fourth, data transference obtains spatial information by converting digital information through specific software such as GIS. This method is highly efficient as it can handle a wide range of spatial information at a given place [4,7], but it does not obtain information when there is no spatial data in a target area [4].

These methods for spatial information acquisition include a variety of detailed techniques, and the most representative techniques in terms of cost and time efficiency may be Computer Aided Design (CAD) file conversion [4,9], image warping [10,11] of as-built drawings, and Global Network Satellite System (GNSS) mapping [1,2]. The CAD file conversion technique utilizes digital as-built drawings with relatively up-to-date spatial information [8], and thus, it is easy to store, utilize and convert data on forest roads. The image warping technique is used for digital mapping of forest roads by calibrating image data on the as-built drawing based on the position coordinates established by GNSS surveys [11]. GNSS surveys are very useful in forest areas with a high-degree of roughness (i.e., complex topography, geology, and vegetation), and can produce relatively accurate and inexpensive thematic maps within a relatively small area $[2,3]$.

Several studies $[1,2,8,9]$ evaluated the accuracy of spatial information gathered from forest roads and compared the positional accuracy of GNSS field data collected from forest routes and its availability. GNSS mapping is considered a cost-effective tool to help manage and maintain forest road systems [1]. Kim et al. [9] produced digital maps of forest road networks using GNSS field data, satellite imagery, ortho-aerial photographs, and digital photogrammetry, and evaluated the positional accuracy and work efficiency. They concluded that the digital photogrammetric method was the most accurate although it required the greatest amount of time. In addition, advances in survey equipment, such as Airborne Laser Scanner (ALS) or Terrestrial Laser Scanner (TLS) obtain high-resolution spatial data, but this equipment has the disadvantage of a high cost to obtain data [8]. In recent years, 3D-based surveying technology was introduced to acquire precise and accurate spatial information. Both ALS and Structure from Motion (SfM) processing is being used to acquire spatial data based on a point-cloud for forest resource measurement [8,12]. For example, White et al. [8] measured the accuracy of log-haul roads and terrain using Light Detection and Ranging (LiDAR), generated Digital Terrain Models (DEMs), and evaluated the availability of Airborne LiDAR for spatial information on forest road and terrain. 
The accuracy of digital maps can be evaluated by point-to-point positional comparisons [1,13] or shape similarity [14,15]. In order to evaluate the positional accuracy of a point object, the most commonly-used method is to determine the error (e.g., Root Mean Square Error (RMSE)) using a specific point or a corresponding point $[1,13]$. However, it is difficult to evaluate the accuracy and error range of features in linear or polygon form [14,16]. Therefore, many studies used RMSE of two- or three-dimensional coordinates to compare the geographical accuracy of linear objects such as roads $[1,8,9]$. In addition, methods have been developed to accurately evaluate the shape similarity or matching of linear or polygonal objects. This is similar to a buffering analysis that compares shape differences by evaluating the area size and shape of a polygon created by overlaying two objects $[14,15]$ and the turning function by plotting the rotation angles and lengths of polygonal chains $[17,18]$. However, previous studies have only investigated the positional accuracy of each tool and none have compared both the positional accuracy and shape similarity of forest roads by digital mapping techniques, such as CAD file conversion, image warping, and GNSS mapping techniques. Without investigating these, the accuracy of these tools may not be fully evaluated.

Consequently, the aim of this study is to evaluate the horizontal accuracy, shape similarity, and cost of creating forest road maps using different mapping techniques. Specifically, we (i) made digital forest road maps using three techniques: (1) CAD file conversion, (2) image warping, and (3) GNSS mapping, and (ii) compared their accuracy, shape similarity time, and cost. The results of this study will contribute to improved long-term forest road maintenance, and road construction with a digital map of forest roads.

\section{Materials and Methods}

\subsection{Study Area Description}

This study consisted of five forest road routes that were located in Chungcheongnam-do in the Republic of Korea (Figure 1). The length of each route was 1-1.4 km (Table 1) and were a part of the national forest road system of the Korea Forest Service (KFS). This study compares two-dimensional horizontal accuracy and, therefore, forest road sections with a small altitude difference were selected.

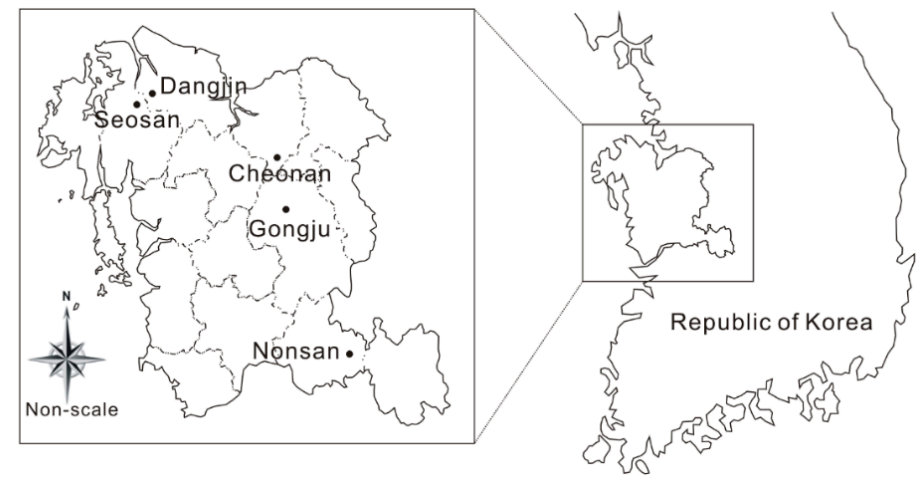

Figure 1. Study site locations.

Table 1. Information on study forest road routes.

\begin{tabular}{|c|c|c|c|c|c|c|c|}
\hline \multirow{2}{*}{ Location } & \multirow{2}{*}{$\begin{array}{l}\text { Length } \\
\text { (m) } 1\end{array}$} & \multirow{2}{*}{$\begin{array}{c}\text { Construction } \\
\text { Year }\end{array}$} & \multicolumn{4}{|c|}{ Altitude (m) } & \multirow{2}{*}{ Forest Type } \\
\hline & & & B.P. ${ }^{2}$ & E.P. ${ }^{3}$ & Max. & Min. & \\
\hline Dangjin & 1417 & 2009 & 147 & 190 & 190 & 143 & Hardwood-forest \\
\hline Seosan & 1275 & 2008 & 116 & 98 & 124 & 97 & Softwood-forest \\
\hline Nonsan & 1012 & 2009 & 151 & 154 & 175 & 151 & Mixed forest \\
\hline Choenan & 1020 & 2007 & 174 & 197 & 197 & 167 & Mixed forest \\
\hline Gongju & 1105 & 2009 & 401 & 412 & 447 & 397 & Mixed forest \\
\hline
\end{tabular}




\subsection{Mapping Techniques of Forest Roads}

Digital road maps were made using three road-mapping techniques: (i) CAD file conversion, (ii) image warping, and (iii) GNSS mapping. Once mapping was complete, each method had their horizontal accuracies compared to the field-survey maps (i.e., reference maps) that were considered the most accurate (Figure 2, Figure S1). Development of a reference map was done with a SET530R Total Station (SOKKIA, Seoul, Korea) with an error range of $\pm 2 \mathrm{~mm}$. This technique was used to measure the centerline and outline of the study routes. Reference maps were made using Power Comms [19] and AutoCAD [20].

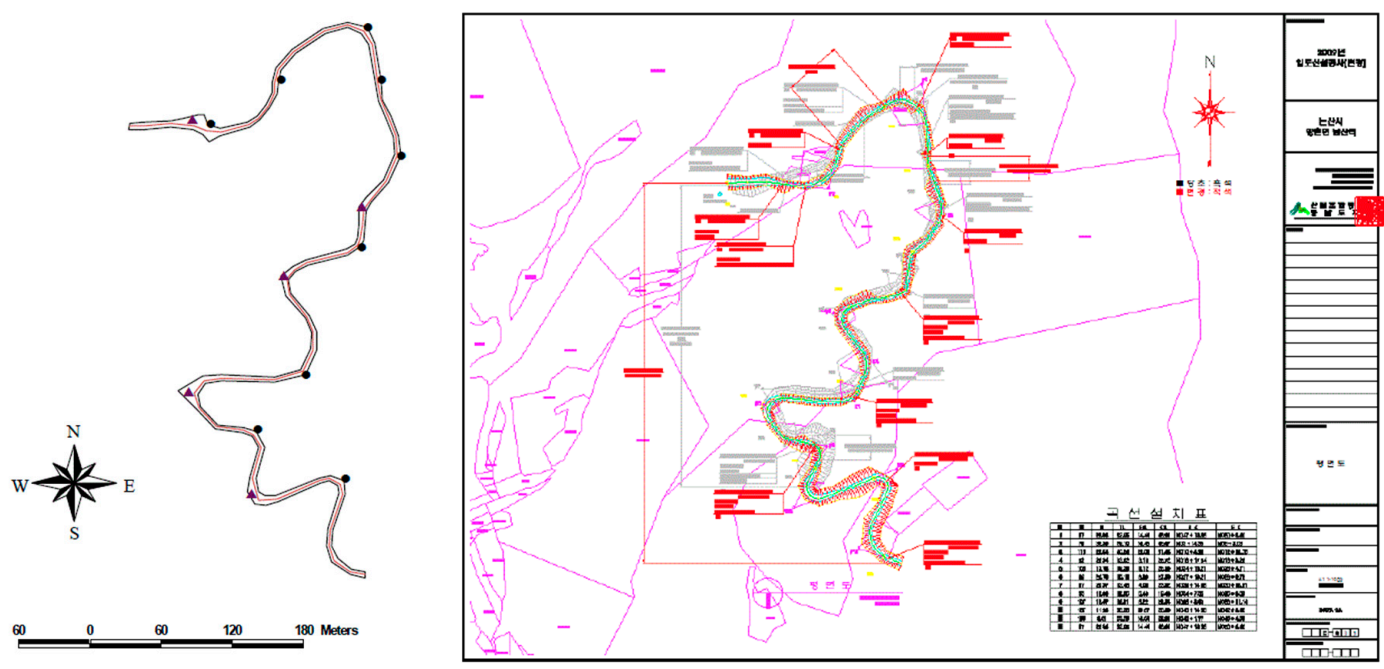

Figure 2. Examples of the reference map using the total station (left, ${ }^{\wedge} \mathrm{GCP}$, - centerline of forest road) and conversion from the as-built drawing file to DXF file format (right).

The CAD file conversion technique created road maps using the as-built drawings, which comprised of CAD files without further enhancement (Figure 2). Only the objects related to the route were selected and converted to a CAD data file format (Drawing Exchange Format, DXF).

The CAD file conversion technique created road maps by scanning the as-built drawing files. The image warping technique used as-built drawings based on Ground Control Point (GCP). Image warping scanned and enhanced the blueprints from as-built drawings using the affine (linear) transformation [21] and image warp feature of ESRI ArcViewß3.2 [22]. We found that the affine transformation worked best for the image warping (Figure 3).
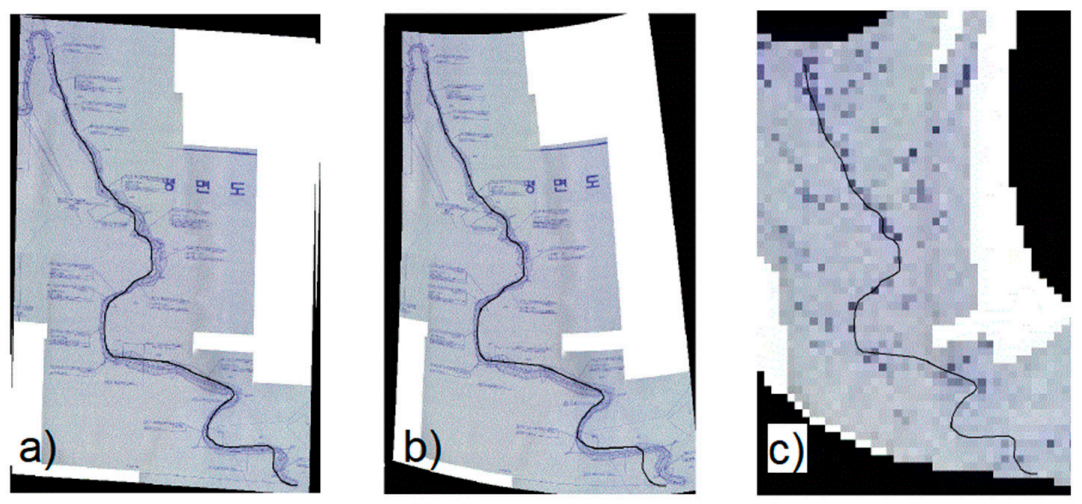

Figure 3. Examples of image warping using blueprints and GCP: (a) 1st transformation (linear), (b) 2nd transformation, (c) 3rd transformation. The image warping effect decreases when using the 2 nd and 3rd transformations. 
The GNSS mapping used a Pro-XRS (Trimble Corp., Sunnyvale, CA) to acquire route data that was downloaded and converted to the road maps using Pathfinder Office software [23] (Figure 4).

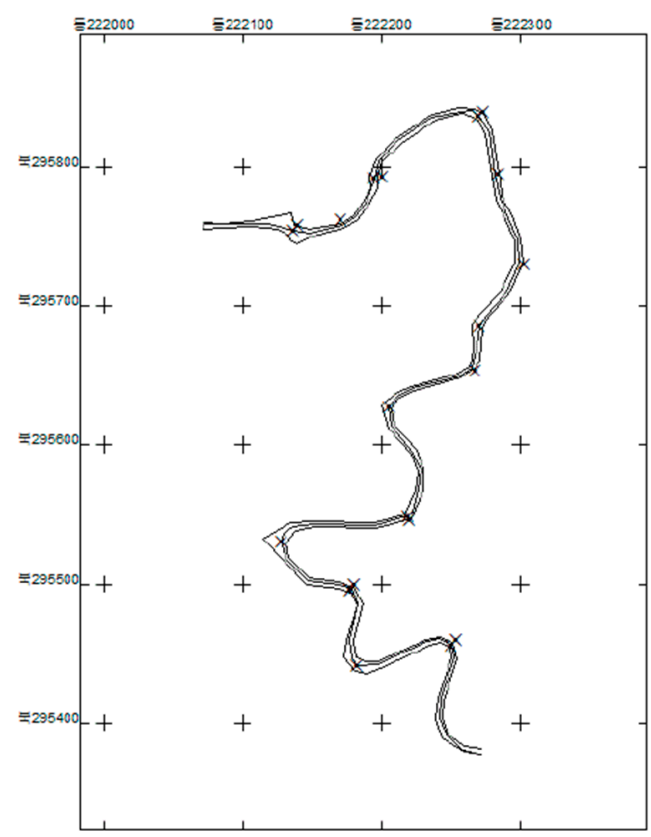

Figure 4. An example of GNSS mapping.

\subsection{Comparison of the Horizontal Accuracy and Shape Similarity between Three Mapping Techniques}

Four methods were used to evaluate the horizontal accuracy and shape similarity between the three mapping techniques: (1) point-correspondence [13,24,25], (2) buffering analysis [14,15], (3) shape index [26], and (4) turning function [17,27]. We only investigated the horizontal positional accuracy because that is more important than vertical accuracy, since most maps are shown in the horizontal plane.

\subsubsection{Point-Correspondence}

Point-correspondence accuracy is based on the corresponding point to evaluate a difference between the same points using different techniques. An error is determined using Root Mean Square Error (RMSE) based on the deviation between the two points on the $x$ - and $y$-axes $[13,24,25]$. Therefore, a single RMSE on the plane position can be calculated as follows:

$$
\text { rmse }=\sqrt{\left(r m s e_{x}\right)^{2}+\left(r m s e_{y}\right)^{2}}
$$

where:

rmse $_{x}$ is root mean square error of $\mathrm{x}$-axis, rmse $_{y}$ is root mean square error of $\mathrm{y}$-axis.

We selected 49 corresponding points from the five forest road routes and calculated RMSE values by the mapping technique and study site location. The results were statistically analyzed with a two-tailed t-test using a significance level of 0.01 .

\subsubsection{Buffering Analysis}

It is very difficult to define the accuracy of vector data on unequal locations. Buffering methods are used to compare the accuracy of linear objects that do not correspond to each other [14,15]. We buffered 
the reference route at $2 \mathrm{~m}$ intervals, calculated the buffer width using $95 \%$ of the comparable routes, and compared the buffer widths by the mapping technique.

\subsubsection{Shape Index}

Buffering methods evaluate map accuracy through proximity to lines that do not correspond to each other, but the similarity of shapes cannot be determined. Therefore, it is necessary to further compare the similarity of the shapes of the study routes. Shape index is a method of comparing shapes as an index used to calculate the shape ratio. It is expressed as a ratio of area to circumference length [28]. Shape index was calculated for the polygon generated from the buffering analysis. If the shape was circular, the shape index is one (i.e., the small perimeter in a given internal area). If the shape was square, the shape index is 1.13 . The shape index can be expressed as follows [15]:

$$
D_{i}=\frac{p}{2 \sqrt{A \pi}}
$$

where:

$D_{i}$ : shape index,

$p$ : perimeter $(\mathrm{m})$,

A: Area $\left(\mathrm{m}^{2}\right)$.

\subsubsection{Turning Function Analysis}

Turning function analysis is another method to compare the shape differences of objects, by plotting the rotation angles and lengths of polygonal chains $[17,18]$. It typically uses a graph that has the $x$-axis as the length of the comparing object and the $y$-axis as the rotation angle. Thus, the $x$-axis depends on the chain length. The y-axis value increases as the rotation angle of the chain increases, and decreases as the rotation angle decreases.

We had one reference map and three maps from each mapping technique. Therefore, the total lengths of polygonal chains might vary depending on the mapping technique. In order to only compare shape similarities, the total length was set to $100 \%$ and the ratio of each polygonal chain length was calculated $[17,27]$. After that, the rotational angles and ratios of polygonal chain lengths were plotted (Figure 5a,b). The shape similarities were compared by calculating the area difference between the graphs plotted from the reference map and three mapping techniques (Figure $5 \mathrm{c}$ ). The area difference between two graphs is a relative value, and therefore a lower value indicates a higher shape similarity.

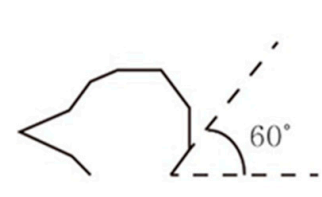

(a)

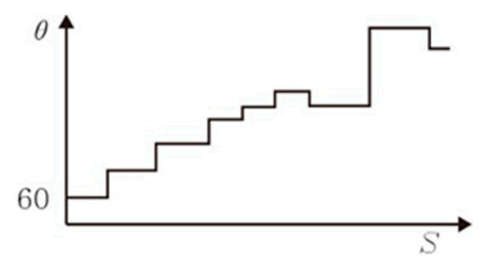

(b)

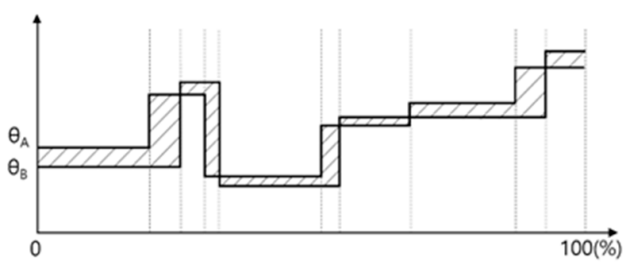

(c)

Figure 5. Turning function analysis: (a) calculating rotational angles and polygonal chain length, (b) plotting rotational angles and ratios of polygonal chain lengths, and (c) comparing shape similarities by calculating the area difference.

\subsubsection{Estimation of Map-Making Time and Cost}

The working time of the three mapping techniques was estimated by measuring the time spent on the field survey and digital map-making of the five road routes. We determined the labor cost [29] and 
work time to estimate the total cost. Worktime and cost are presented as an average cost per unit road length (US dollar $/ \mathrm{km}$ ).

\section{Results}

There were differences in route length between the maps created when using the total station and three mapping techniques (Table 2). The Total Station data indicated that the length of the study area was $5662 \mathrm{~m}$. This was shorter than the lengths produced by both the CAD file and image warping, but very similar to GNSS mapping.

Table 2. Comparisons of the study route length by mapping technique.

\begin{tabular}{cccccc}
\hline Area & No. of IP ${ }^{\mathbf{1}} \mathbf{( e a )}$ & Total Station (m) & $\begin{array}{c}\text { CAD File } \\
\text { Conversion (m) }\end{array}$ & $\begin{array}{c}\text { Image } \\
\text { Warping (m) }\end{array}$ & $\begin{array}{c}\text { GNSS } \\
\text { Mapping (m) }\end{array}$ \\
\hline Dangjin & 11 & 1398 & $1417(\triangle 19)$ & $1438(\Delta 40)$ & $1396(\nabla 2)$ \\
Seosan & 19 & 1209 & $1275(\triangle 66)$ & $1280(\Delta 71)$ & $1211(\triangle 2)$ \\
Nonsan & 12 & 985 & $1012(\triangle 27)$ & $1040(\Delta 55)$ & $986(\Delta 1)$ \\
Cheonan & 13 & 1048 & $1020(\nabla 28)$ & $1123(\Delta 75)$ & $1047(\nabla 1)$ \\
Gongju & 23 & 1022 & $1105(\triangle 83)$ & $1101(\Delta 79)$ & $1027(\triangle 5)$ \\
Total & 78 & 5662 & $5829(\triangle 167)$ & $5982(\triangle 320)$ & $5667(\triangle 5)$ \\
\hline
\end{tabular}

${ }^{1}$ Intermediate point.

\subsection{Point-Correspondence}

After analyzing 49 points along the surveyed routes, the average RMREs from CAD file conversion, image warping, and GNSS mapping were $13.53 \mathrm{~m}, 7.13 \mathrm{~m}$, and $1.28 \mathrm{~m}$ respectively, indicating that the GNSS mapping accuracy is the highest (Table 3). In particular, CAD file conversion had 10.4 times the mean and 8.56 times the standard deviation of the RMSE as compared to GNSS mapping.

Table 3. Comparison of point-correspondence results (RMSE) by mapping technique.

\begin{tabular}{|c|c|c|c|c|c|c|c|c|c|c|c|c|}
\hline \multirow{2}{*}{ Mapping Technique } & \multicolumn{2}{|c|}{ Total } & \multicolumn{2}{|c|}{ Dangjin } & \multicolumn{2}{|c|}{ Seosan } & \multicolumn{2}{|c|}{ Nonsan } & \multicolumn{2}{|c|}{ Cheonan } & \multicolumn{2}{|c|}{ Gongju } \\
\hline & Mean & SD $^{1}$ & Mean & SD & Mean & SD & Mean & SD & Mean & SD & Mean & SD \\
\hline CAD file conversion & $13.35^{\mathrm{a}}$ & 7.36 & $14.78^{c}$ & 9.38 & $14.51^{\mathrm{c}}$ & 6.25 & $11.04^{\mathrm{c}}$ & 4.52 & $13.07^{\mathrm{c}}$ & 6.86 & $14.01^{c}$ & 7.68 \\
\hline $\begin{array}{l}\text { GNSS } \\
\text { mapping }\end{array}$ & $1.28^{c}$ & 0.86 & $1.99^{\mathrm{a}}$ & 0.63 & $0.81^{\mathrm{a}}$ & 0.48 & $1.08^{\mathrm{a}}$ & 0.64 & $1.16^{\mathrm{a}}$ & 0.82 & $1.36^{\mathrm{a}}$ & 1.00 \\
\hline$F$ & \multicolumn{2}{|c|}{79.89} & \multicolumn{2}{|c|}{11.10} & \multicolumn{2}{|c|}{24.28} & \multicolumn{2}{|c|}{22.08} & \multicolumn{2}{|c|}{17.08} & \multicolumn{2}{|c|}{14.14} \\
\hline
\end{tabular}

${ }^{1}$ SD: Standard deviation. Superscript letters $(a, b$, and c) next to means represent significant difference at $\alpha=0.01$.

The positional accuracy (RMSE) of the image warping is about $47 \%$ as compared to the CAD file conversion, indicating that image warping is more accurate than the CAD file conversion. For all five study routes, the RMSEs were significantly different.

\subsection{Buffering Analysis}

In the buffering analysis, overlaying the CAD file conversion maps on top of the reference maps resulted in a buffer width of $24 \mathrm{~m}$, and greater than $95 \%$ of routes being included in the buffer range. Buffer widths, when overlapping 95\% of the routes, were as follows: Gongju (16 m), Dangjin (18 m), Nonsan (18 m), Cheonsan (22 m), and Seosan (24 m) (Figure S2). Overlaying the image warping and reference maps indicated that with a buffer width of $18 \mathrm{~m}$, more than $95 \%$ of the routes were included in the buffer range (Figure S3). Buffer width, when overlapping 95\% of the routes, were as follows: Nonsan (10 m), Dangjin (12 m), Cheonsan (12 m), Gongju (18 m) and Seosan (18 m). GNSS mapping allowed the use of the narrowest buffer width (Figure 6). On average, more than 95\% of the routes were included in the buffer range of the reference map when the buffer width was $1.5 \mathrm{~m}$. When the all routes $(100 \%)$ were included, buffer widths were $4 \mathrm{~m}$ (Figure S4). 


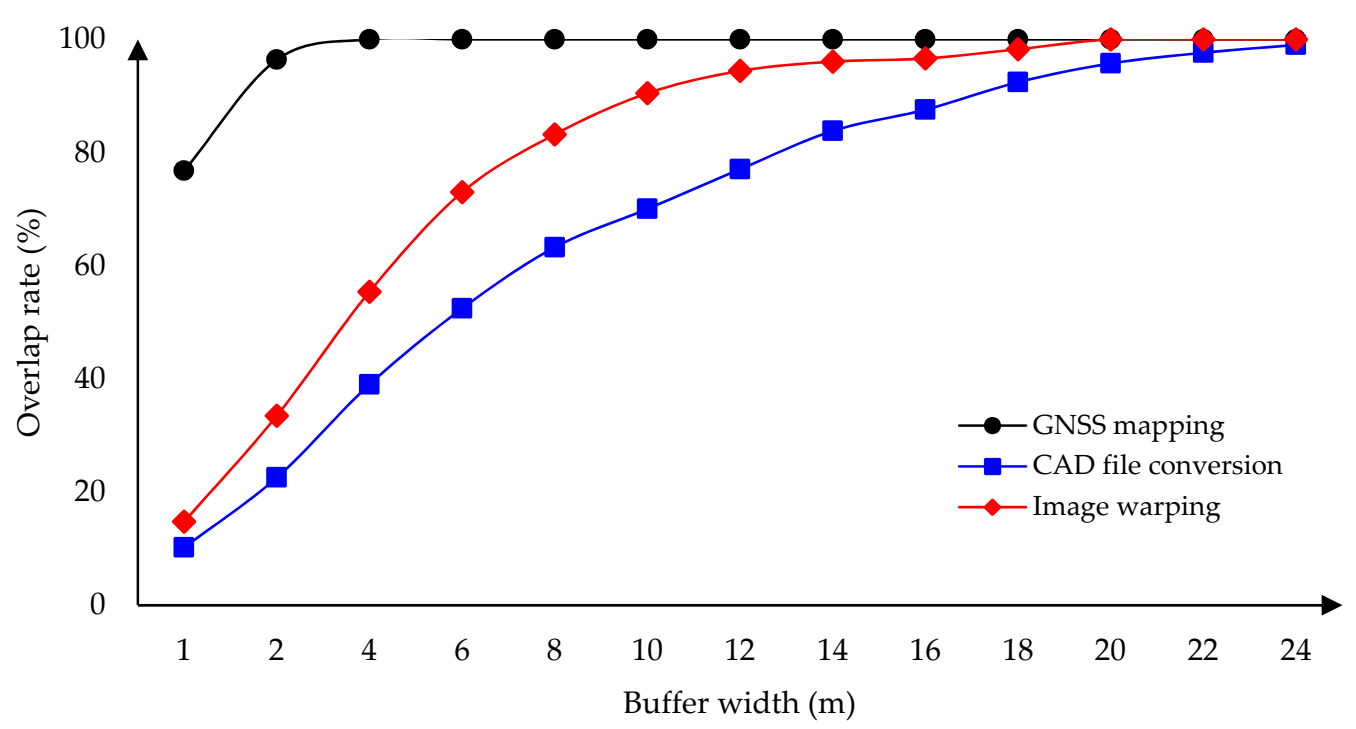

Figure 6. Results of buffering analysis by mapping technique. The overlapping rates were average values.

\subsection{Shape Index}

The shape index of the converted CAD file was 6.5-7.4, image warping was 7.2-10.8, and GNSS mapping was 19.6-28.9 (Table 4). As previously noted, when the shape of the measured polygon is circular the shape index is 1 . The index increases when the shape elongates (e.g., becomes bar-shaped) [26]. Therefore, a higher index indicates that the shape is closer to the reference map. In general, polygons from GNSS mapping results in a shape index greater than 20, indicating narrow and long shapes more closely resembling the reference map. In contrast, the shape indices from the CAD file conversion and image warping methods resulted in shape indices that were generally less than 10 , indicating broader shapes and less shape similarity to the reference map.

Table 4. The shape index by mapping technique.

\begin{tabular}{ccccc}
\hline Route Location & Mapping Technique & Area $\left.\mathbf{( m}^{\mathbf{2}}\right)$ & Length $\mathbf{( m )}$ & Shape Index \\
\hline \multirow{3}{*}{ Dangjin } & CAD file conversion & 11,715 & 2839.7 & 7.4 \\
& Image warping & 5548 & 2852.8 & 10.8 \\
& GNSS mapping & 1624 & 2800.0 & 19.6 \\
\multirow{3}{*}{ Seosan } & CAD file conversion & 11,884 & 2506.6 & 6.5 \\
& Image warping & 6733 & 2503.9 & 8.6 \\
& GNSS mapping & 557 & 2422.0 & 28.9 \\
Nonsan & CAD file conversion & 6040 & 2019.4 & 7.3 \\
& Image warping & 3517 & 2037.1 & 9.7 \\
& GNSS mapping & 554 & 1974.0 & 23.7 \\
Cheonan & CAD file conversion & 6942 & 2123.8 & 7.2 \\
& Image warping & 4582 & 2194.1 & 9.1 \\
& GNSS mapping & 479 & 2099.0 & 27.0 \\
Gongju & CAD file conversion & 8612 & 2165.4 & 6.6 \\
& Image warping & 7169 & 2164.2 & 7.2 \\
& GNSS mapping & 681 & 2042.0 & 22.1 \\
\hline
\end{tabular}

\subsection{Turning Function Analysis}

Differences in mapped area size among the three mapping techniques as compared to the reference map were as follows: CAD file conversion $(8661-27,845)$, image warping $(7972-26,256)$, and GNSS mapping (2814-4949) (Table 5, Figure 7, Figures S5-S8). Overall, the mapped area differences were 
lowest using GNSS mapping, followed by image warping, and CAD file conversion. Our results indicate that the GNSS mapping produced maps that were most similar to the reference map.

Table 5. Area size difference in turning function analysis between the three mapping techniques and reference map.

\begin{tabular}{cccc}
\hline \multirow{2}{*}{ Route Location } & \multicolumn{3}{c}{ Mapping Technique } \\
\cline { 2 - 4 } & CAD File Conversion & Image Warping & GNSS Mapping \\
\hline Dangjin & 8661 & 7972 & 3357 \\
Seosan & 19,633 & 16,984 & 3230 \\
Nonsan & 14,211 & 13,994 & 2814 \\
Cheonan & 23,588 & 19,331 & 4130 \\
Gongju & 27,845 & 26,256 & 4949 \\
\hline
\end{tabular}

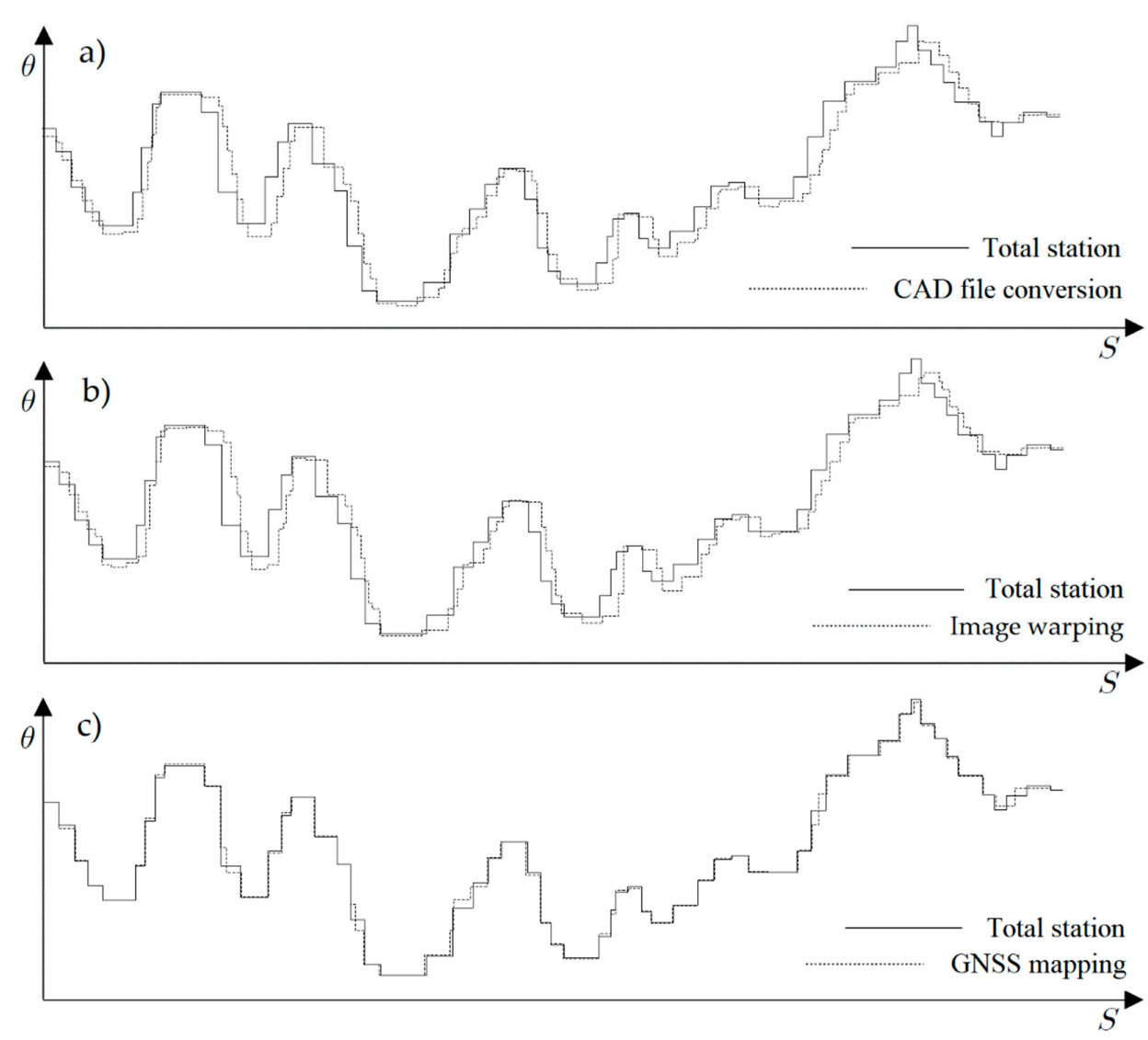

Figure 7. Example of turning function analysis between the three mapping techniques and reference map on Nonsan: (a) CAD file conversion, (b) image warping, and (c) GNSS mapping.

\subsection{Estimation of Map-Making Time and Cost}

The working time of three mapping techniques, inclusive of field survey and map-making, were $236 \mathrm{~min} / \mathrm{km}$ for GNSS mapping, $180 \mathrm{~min} / \mathrm{km}$ for image warping, and $99 \mathrm{~min} / \mathrm{km}$ for CAD file conversion. Production costs to create a digital map were $\$ 139.64 / \mathrm{km}$ for GNSS, $\$ 81.84 / \mathrm{km}$ for image warping, and $\$ 40.90 / \mathrm{km}$ for CAD file conversion, indicating that CAD file conversion was the most cost-efficient (Table 6). In addition, the working time to produce the reference map using the total station was $315 \mathrm{~min} / \mathrm{km}$ (195 $\mathrm{min} / \mathrm{km}$ for on-site survey and $120 \mathrm{~min} / \mathrm{km}$ for map-making work), and the total cost was $\$ 180.66 / \mathrm{km}$. 
Table 6. Time study and cost analysis of forest road digital map by three mapping techniques.

\begin{tabular}{ccccc}
\hline \multirow{2}{*}{ Mapping Technique } & \multicolumn{2}{c}{ Average Working Time $\mathbf{( m i n / \mathbf { k m } )}$} & \multirow{2}{*}{$\begin{array}{c}\text { Production Cost } \\
\text { (US\$/km) }{ }^{\mathbf{3}}\end{array}$} \\
\cline { 2 - 4 } & Field Survey $^{\mathbf{1}}$ & Map-making $^{\mathbf{2}}$ & Total & 40.90 \\
\hline CAD file conversion & - & 99 & 99 & 81.84 \\
Image warping & 31 & 149 & 180 & 139.64 \\
GNSS mapping & 175 & 61 & 236 & 180.66 \\
Total station & 195 & 120 & 315 & \\
\hline
\end{tabular}

${ }^{1}$ Conducted by one experienced technician (\$175.47/day) and one beginner technician (\$138.25/day). ${ }^{2}$ Conducted by one expert technician (\$198.22/day). ${ }^{3}$ Converted from Korean Won ( $\left.\$\right)$ based on 2018 currency exchange rate [30].

\section{Discussion}

Map accuracy is one metric for determining the best mapping method and, for our study, map accuracy followed the trend (from greatest to smallest) of GNSS, image warping, and CAD file conversion. However, there are several error-sources inherent within spatial data that should be addressed before making a digital map using GIS or CAD programs. In the forest environment, the constraints of surveying include difficult terrain and trees taller than the user, but GNSS is primarily used because of convenience and efficiency $[1,2,31]$. Many studies have shown, however, that the accuracy of GPS-based surveying has an error range of 1-88 $\mathrm{m}[2,8]$ and depends on the user and/or receiver environment. In particular, the failure to receive satellite signals because they are blocked by a forest canopy or hilly terrain during forest road route surveys is one possibility of user error [1]. Therefore, in order to increase the accuracy of GPS surveying, it is necessary to reduce the influence of the forest canopy and terrain, to have enough time to receive satellite signals, and to educate and train users to reduce human error [1,32]. Scanning and data transferring methods such as image warping and CAD file conversion might also have an error of positional accuracy due to the data itself. For example, during road construction changes made in the route are often not reflected in the as-built drawings. After completion of road construction, the as-built drawings are made using blueprints rather than conducting field surveys again. We considered data inaccuracy as a part of the overall methodological errors, due to lack of detailed field survey data after the road construction. Future studies are needed to further assess the different errors associated with image warping and CAD file conversion.

In many forest studies the positional accuracy was evaluated by measuring the difference between a known reference point and the corresponding points, and then calculating the position error (e.g., RMSE). However, it is difficult to compare the morphological differences of line segments $[14,16]$ because midand endpoints in a line segment can contain disproportionate error terms [33]. Furthermore, since linear networks, such as roads, often do not have adequate comparison points, it may be more appropriate to simply compare features within a road network to a more accurate, linear reference $[14,15]$ or to internal digital geometry $[24,34,35]$.

Shape matching is important for comparing accuracy and similarity [18], especially for a line or polygon segment such as the centerline of roads, railways, shorelines, and boundaries of natural phenomena [15]. Thus, we used the highly accurate reference map to compare the positional accuracy and shape similarity of the other mapping methods by using point-correspondence and buffering analysis. The results from both methods showed that GNSS mapping was the most accurate technique. However, this method also takes more time and has a greater associated cost to collect field data and construct maps as compared to the other two techniques. For the CAD file conversion technique, the only time requirement was to input or edit locations and information on the forest road routes and facilities without the need for a field survey. Therefore, it took $42 \%$ less field and mapping time and had $27 \%$ less cost as compared to GNSS. Image warping took more time than the CAD file conversion because it required a field survey to obtain the GCP and additional processing time to scan the drawings. 
This study reports high GNSS time and costs and is similar to findings of previous studies. Kim et al. [9] reported it took $17 \mathrm{~h}$ to measure $35 \mathrm{~km}$ of forest road routes using a GPS receiver, while it took $6 \mathrm{~h}$ using aerial imagery. Furthermore, Ömer \& Ayhan [7] reported that the production cost of GNSS mapping for spatial information $(\$ 17.22 /$ ha) was about 3.6 times that of the digitizing method (\$4.79/ha).

LiDAR is a widely used remote sensing technology that can be used as a road mapping method $[8,36]$. In Korea, road map-making using LiDAR costed $\$ 11.05 /$ ha for 1:1000 map and $\$ 7.10 /$ ha for 1:5000 map [30,37], which are less than GNSS but greater than the digitizing method [7]. Future studies are needed to assess more up-to-date map-making technologies for forest road, such as LiDAR and 3D-based geographical information using point-cloud.

If higher map-making accuracy is required, then there will be associated increase in mapping costs. Similarly, if mapping funds are limited, map accuracy will be lower [9]. Therefore, it is necessary to make decisions regarding the trade-off between accuracy and cost, while considering the demand and purpose of maps. GNSS mapping techniques are most suitable for small-scale mapping or short-haul routes where a small error range is required (e.g., less than $1 \mathrm{~m}$ ). CAD file conversion can be accurate because it converts as-built drawings with high accuracy into a map. However, this study found that the data did not match well with the reference map and the technique was less accurate than GNSS mapping. In Korea, more efforts should be made to improve the accuracy of the as-built drawings and CAD file conversion. Due to their lower level of accuracy, CAD file conversion is recommended for making a large-scale road inventory at the regional or national level. Due to low accuracy, the image warping technique can also be considered for large-scale mapping or long-distance routes, such as $1: 25,000$ or greater.

This study compared the horizontal accuracy of different mapping techniques in forested areas in Korea. Considering that most maps are shown in the horizontal plane, we did not include vertical accuracy in this study. However, Korea has steep terrains [38], and vertical accuracy might be of importance and is worth future investigations.

\section{Conclusions}

This study compared the horizontal accuracy, shape similarity, and production cost of creating forest road maps using different mapping techniques (i.e., CAD file conversion, image warping, and GNSS mapping) to determine the most effective methods to produce digital forest road maps. We conclude that GNSS mapping is the best technique when considering high accuracy and shape similarity. GNSS mapping, however, requires a substantial amount of time and considerable costs to produce digital maps. Therefore, GNSS mapping should be used for small-scale mapping or short-haul routes that require a small error range, while CAD file conversion and image warping can be used for large-scale mapping. CAD file conversion has been shown to be relatively accurate, but our data did not match well with the reference map and were less accurate than GNSS mapping. Since this method is less expensive and can produce maps suitable for long distances (large areas), greater effort should be made to improve the accuracy of the CAD file conversion in Korea. In addition, the accuracy of GPS surveying for GNSS mapping can be increased by educating and training users on how to reduce the influence of the forest canopy and terrain, while ensuring that there is enough time to receive satellite signals.

Effective forest management and harvest operations require precise spatial information. This study contributes knowledge about how to improve forest road mapping accuracy. There are increasing interests and needs (e.g., earthwork, cut and fill) for 3D-based geographical information using point-cloud. In the future, it will be necessary to make 3D road maps from point-cloud based data and to utilize this to develop an effective and efficient road inventory for large aerial extent mapping projects in mountainous areas. 
Supplementary Materials: The following are available online at http://www.mdpi.com/1999-4907/10/5/452/s1, Figure S1: Reference map of study road routes using total station: (a) Dangjin, (b) Cheoan, (c) Seosan, and (d) Gongju ( ${ }^{\wedge}$ GCP, - Center line of forest road), Figure S2: Example of buffering analysis between the CAD file conversion and reference map: (a) Dangjin, buffer width is $6 \mathrm{~m}(40.1 \%)$, (b) Dangjin, buffer width is $16 \mathrm{~m}(96.1 \%)$, (c) Seosan, buffer width is $10 \mathrm{~m}(54.0 \%)$, (d) Seosan, buffer width is $24 \mathrm{~m}(95.1 \%)$, (e) Nonsan, buffer width is $8 \mathrm{~m}(69.7 \%)$, (f) Nonsan, buffer width is $18 \mathrm{~m}(95.0 \%)$, (g) Cheonan, buffer width is $6 \mathrm{~m}(62.8 \%)$, (h) Cheonan, buffer width

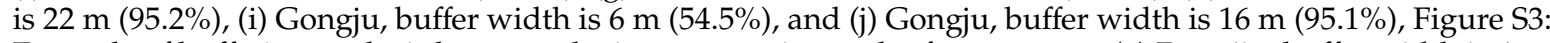
Example of buffering analysis between the image warping and reference map: (a) Dangin, buffer width is $4 \mathrm{~m}$ $(62.3 \%)$, (b) Dangjin, buffer width is $12 \mathrm{~m}(98.9 \%)$, (c) Seosan, buffer width is $6 \mathrm{~m}(65.7 \%)$, (d) Seosan, buffer width is $18 \mathrm{~m}(96.3 \%)$, (e) Nonsan, buffer width is $4 \mathrm{~m}(58.8 \%)$, (f) Nonsan, buffer width is $10 \mathrm{~m}(97.8 \%)$, (g) Cheonan, buffer width is $4 \mathrm{~m}(51.3 \%)$, (h) Cheonan, buffer width is $12 \mathrm{~m}(96.8 \%)$, (g) Gongju, buffer width is $2 \mathrm{~m}(46.5 \%)$, and (h) Gongju, buffer width is $18 \mathrm{~m}(95.0 \%)$, Figure S4: Example of buffering analysis between the GNSS mapping and reference map: (a) Dangjin, buffer width is $2 \mathrm{~m} \mathrm{(85 \% ),} \mathrm{(b)} \mathrm{Dangjin,} \mathrm{buffer} \mathrm{width} \mathrm{is} 4 \mathrm{~m} \mathrm{(100 \% ),} \mathrm{(c)} \mathrm{Seosan,}$ buffer width is $1 \mathrm{~m}(91 \%)$, (d) Seosan, buffer width is $4 \mathrm{~m} \mathrm{(100 \% ),} \mathrm{(e)} \mathrm{Nonsan,} \mathrm{buffer} \mathrm{width} \mathrm{is} 1 \mathrm{~m}(78 \%)$, (f) Nonsan, buffer width is $4 \mathrm{~m}(100 \%)$, (g) Cheonan, buffer width is $1 \mathrm{~m}(93 \%)$, (h) Cheonan, buffer width is $2 \mathrm{~m}(100 \%)$, (i) Gongju, buffer width is $1 \mathrm{~m}(73 \%)$, and (j) Gongju, buffer width is $4 \mathrm{~m}(100 \%)$, Figure S5: Example of turning function analysis between the three mapping techniques and the reference map on Dangjin: (a) GNSS mapping, (b) as-built drawing, and (c) image warping, Figure S6: Example of turning function analysis between the three mapping techniques and the reference map on Seosan: (a) GNSS mapping, (b) as-built drawing, and (c) image warping, Figure S7: Example of turning function analysis between the three mapping techniques and the reference map on Cheonan: (a) GNSS mapping, (b) as-built drawing, and (c) image warping, Figure S8: Example of turning function analysis between the three mapping techniques and the reference map on Gongju: (a) GNSS mapping, (b) as-built drawing, and (c) image warping.

Author Contributions: J.-W.L. and M.K. conceived and designed the experiment; M.K. and H.K. performed the experiment and analyzed the data; and H.K., J.I.S., and H.R. wrote the paper.

Funding: This study was carried out with the support of "R\&D Program for Forest Science Technology (Project No. 2018125B10-1920-AB01)" provided by Korea Forest Service (Korea Forestry Promotion Institute).

Acknowledgments: The authors would like to thank Deborah Page-Dumroese for her reviews and comments on this manuscript.

Conflicts of Interest: The authors declare no conflict of interest.

\section{References}

1. Abdi, E.; Sisakht, L.; Goushbor, L.; Soufi, H. Accuracy assessment of GPS and surveying technique in forest road mapping. Ann. For. Res. 2012, 55, 309-317.

2. Talebi, M.; Majnounian, B.; Ehsan, A.; Tehrani, F.B. Development a GIS database for forest road management in Arasbaran forest, Iran. For. Sci. Technol. 2015, 11, 27-35. [CrossRef]

3. Murphy, P.N.C.; Ogilvie, J.; Castonguay, M.; Zhang, C.F.; Meng, F.R.; Arp, P.A. Improving forest operations planning through high-resolution flow-channel and wet-areas mapping. For. Chron. 2008, 84, 568-574. [CrossRef]

4. Yang, S.; Choi, J.; Yu, K. Development of the Digital Map Updating System using CAD Object Extracted from As-Built Drawings. J. Korean Soc. GIS 2009, 17, 13-21, (In Korean with English Abstract).

5. Alizadeh, S.; Majnounian, B.; Darvishsefat, A. Possibility of designing and evaluation of forest road network variants using GIS and field investigation. J. For. Wood Prod. 2011, 63, 399-408.

6. Grigolato, S.; Mologni, O.; Raffaele, C. GIS applications in forest operations and road network planning: An overview over the last two decades. Croat. J. For. Eng. 2017, 38, 175-186.

7. Ömer, M.; Ayhan, C. Accuracy and cost comparison of spatial data acquisition methods for the development of geographical information systems. J. Geogr. Regional Plan. 2009, 2, 235-242. [CrossRef]

8. White, R.A.; Dietterick, B.C.; Mastin, T.; Strohman, R. Forest roads mapped using LiDAR in steep forested terrain. Remote Sens. 2010, 2, 1120-1141. [CrossRef]

9. Kim, T.; Yoon, J.; Woo, C.; Lee, K.; Hong, C. Comparison of methodology and accuracy of digital mapping of forest roads. J. Geogr. Inform. Syst. Assoc. Korea 2005, 13, 195-209. (In Korean)

10. Glasbey, C.; Mardia, K. A review of image warping methods. J. Appl. Stat. 1998, 25, 155-171. [CrossRef]

11. Kim, M.; Kweon, H.; Choi, Y.; Yeom, I.; Lee, J. Evaluation of horizontal position accuracy in forest road completion drawing. Korean J. Agr. Sci. 2010, 37, 471-479, (In Korean with English Abstract).

12. Wallace, L.; Lucieer, A.; Malenovský, Z.; Turner, D.; Vopěnka, P. Assessment of Forest Structure Using Two UAV Techniques: A Comparison of Airborne Laser Scanning and Structure from Motion (SfM) Point Clouds. Forests 2016, 7, 62-78. [CrossRef] 
13. Kagawa, Y.; Sekimoto, Y.; Shibasaki, R. Comparative study of positional accuracy evaluation of line data. In Proceedings of the 20th Asian Conference on Remote Sensing, Hong Kong, China, 22-25 November 1999.

14. Goodchild, M.; Hunter, G. A simple positional accuracy measure for linear features. Int. J. Geogr. Inform. Sci. 1997, 11, 299-306. [CrossRef]

15. Tveite, H.; Langaas, S. An accuracy assessment method for geographical line data sets based on buffering. Int. J. Geogr. Inform. Sci. 1999, 13, 27-47. [CrossRef]

16. Lueng, Y.; Yan, J. A locational error model for spatial features. Int. J. Geogr. Inform. Sci. 1998, 12, $607-620$. [CrossRef]

17. Arkin, E.; Chew, P.; Huttenlocher, D.; Kedem, K.; Mitchel, J. An efficiently computable metric for comparing polygonal shapes. IEEE Trans. Pattern Anal. Mach. Intell. 1991, 13, 209-215. [CrossRef]

18. Velkamp, R.C. Shape matching: similarity measures and algorithms. In Proceedings of the International Conference on Shape Modeling and Applications, Genova, Italy, 7-11 May 2001.

19. SOKKIA KOREA. Available online: https://www.sokkia.co.kr (accessed on 15 August 2010).

20. AutoCAD. Available online: https://www.autodesk.com (accessed on 15 April 2012).

21. Parkhomenko, A.S. Affine transformation. In Encyclopedia of Mathematics; Hazewinkel, M., Ed.; Springer: Berlin, Germany, 2010. Available online: http://www.encyclopediaofmath.org/index.php?title=Affine_ transformation\&oldid=17980 (accessed on 9 September 2018).

22. ESRI. Available online: http://support.esri.com (accessed on 15 August 2010).

23. Trimble Korea. Available online: https://geospatial.trimble.com (accessed on 15 June 2010).

24. Van Niel, T.G.; McVicar, T.R. Experimental evaluation of positional accuracy estimates from a linear network using point-and line-based testing methods. Int. J. Geogr. Inform. Sci. 2002, 16, 459-473. [CrossRef]

25. Ramirez, J.R.; Ali, T. Progress in metrics development to measure positional accuracy of spatial data. In Proceedings of the 21st International Cartographic Conference, Durban, South Africa, 10-16 August 2003; pp. 1763-1772.

26. Forman, R.T.T.; Godron, M. Landscape Ecology; John Wiley and Sons: New York, NY, USA, 1986; pp. 188-189.

27. Velkamp, R.C.; Hagedoorn, M. Shape similarities, properties, and constructions. In Proceedings of the 4th International Conference, Lyan, France, 2-4 November 2000; pp. 467-476.

28. Kim, M.; Ahn, D. Landscape ecological analysis of urban parks -analysis of index of patch shape and the dispersion of patches. J. Korean Inst. Landsc. Archit. 1996, 23, 12-19, (In Korean with English Abstract).

29. Korea Engineering and Consulting Association (KENCA). Available online: https://www.etis.or.kr/webs/ statistics/statistics_board.jsp?leftParam=1\&topParam=3\&boardId=TOTALBBS\&categorygroup2=TB050 (accessed on 6 May 2019).

30. KEB Hanabank. Available online: https://www.kebhana.com (accessed on 14 January 2019).

31. Pirti, A. Accuracy analysis of GPS positioning near the forest environment. Croat. J. For. Eng. 2008, 29, 189-199.

32. Yosimura, T.; Hasegawa, H. Comparing the precision and accuracy of GPS positioning in forested areas. J. For. Res. 2003, 8, 147-152. [CrossRef]

33. Shi, W. A generic statistical approach for modelling error of geometric features in GIS. Int. J. Geogr. Inform. Sci. 1998, 12, 131-143. [CrossRef]

34. Duckham, M.; Drummond, J. Assessment of error in digital vector data using fractal geometry. Int. J. Geogr. Inform. Sci. 2000, 14, 67-84. [CrossRef]

35. Veregin, H. Quantifying positional error induced by line simplification. Int. J. Geogr. Inform. Sci. 2000, 14, 113-130. [CrossRef]

36. Azizi, Z.; Najafi, A.; Sadeghian, S. Forest road detection using LiDAR data. J. For. Res. 2014, 25, 975-980. [CrossRef]

37. Kang, J.M.; Yoon, H.C.; Lee, J.D.; Park, J.K. Analysis of economical efficiency of digital map in production cost by aerial LiDAR surveying. J. KOGSIS 2007, 15, 67-73, (In Korean with English Abstract).

38. Yang, B. Republic of Korea, Nation Map Book II; Ministry of Land, Infrastructure and Transport, National Geographic Information Institute: Suwon, Gyeonggi-do, Korea, 2016. Available online: http://map.ngii.go. $\mathrm{kr} / \mathrm{ms} / \mathrm{pblictn} /$ nationMapBook.do (accessed on 17 September 2018).

(C) 2019 by the authors. Licensee MDPI, Basel, Switzerland. This article is an open access article distributed under the terms and conditions of the Creative Commons Attribution (CC BY) license (http://creativecommons.org/licenses/by/4.0/). 